\title{
TI.116.1
}

\section{TIER Top Stories by Weight}

- PDF: TI.116.1-TIER_Prioritized_Use_Cases.pdf

- Text: TI.116.1-TIER_Prioritized_Use_Cases.txt

\section{More Information}

\begin{tabular}{|l|l|}
\hline Repository ID & TI.116.1 \\
\hline Persistent URL & http://doi.org/10.26869/TI.116.1 \\
\hline Title & TIER Top Stories by Weight \\
\hline Authors & Steve Zoppi, Ann West \\
\hline Sponsor & TIER \\
\hline Review & \\
\hline Status & Legacy \\
\hline Publish Date & April 14, 2015 \\
\hline DOI & 10.26869/TI.116.1 \\
\hline Signature & \\
\hline Deprecated & No \\
\hline Future Review & \\
\hline Supersedes & \\
\hline Format & PDF, Text \\
\hline Related Docs & \\
\hline Development Location & \\
\hline IP Framework & tier \\
\hline Subject Tags & \\
\hline Notes & \\
\hline & \\
\hline
\end{tabular}

